令

SSCL-622

June 1993

Distribution Category: 414

V. V. Parkhomchuk

V. D. Shiltsev

Is Transverse Feedback Necessary for the SSC Emittance Preservation? (Vibration Noise Analysis and Feedback Parameters Optimization)

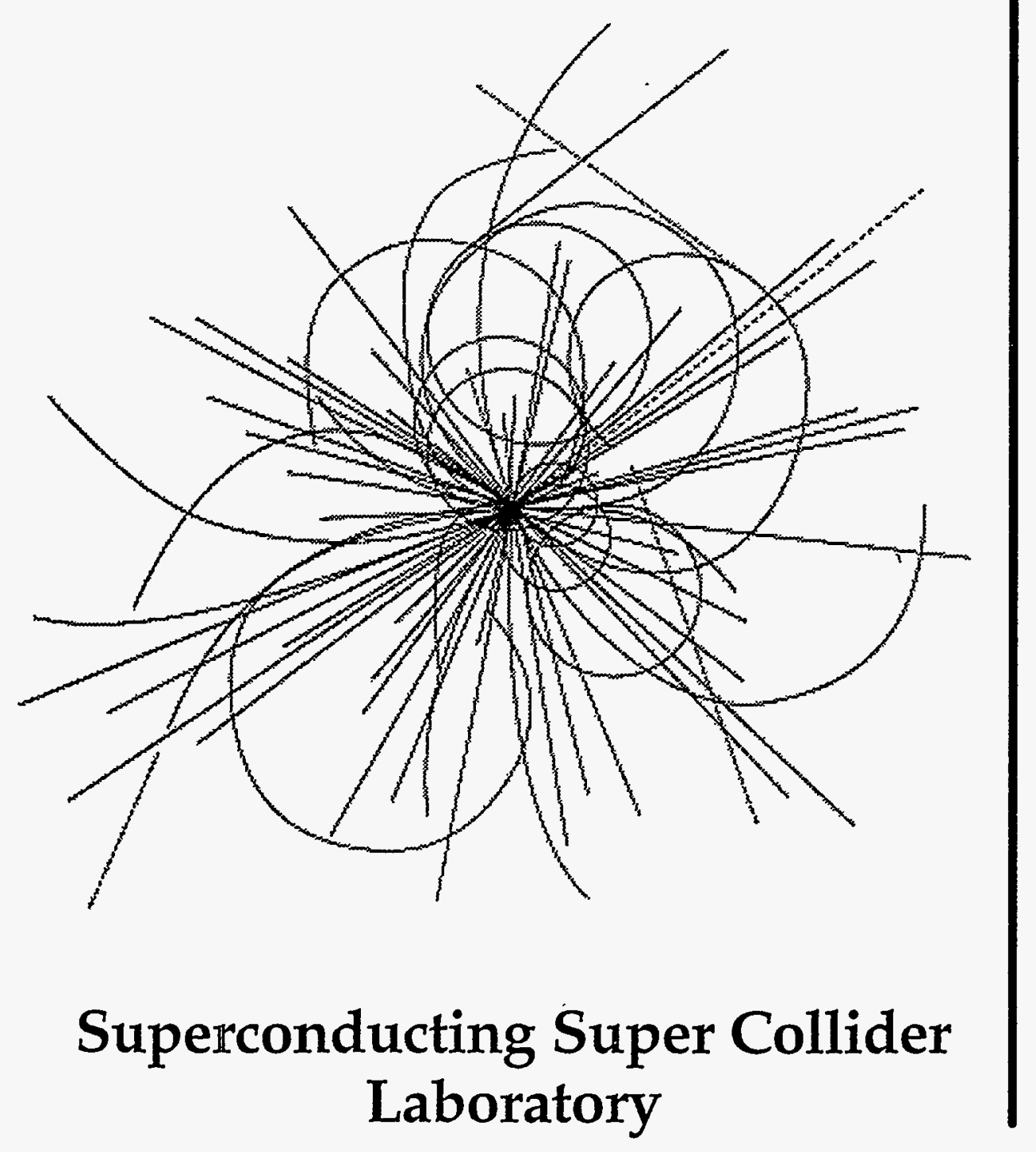




\section{Disclaimer Notice}

This report was prepared as an account of work sponsored by an agency of the United States Govemment. Neither the United States Govemment or any agency thereof, nor any of their employees, makes any warranty, express or implied, or assumes any legal liability or responsibulity" for the accuracy, completeness, or usefulness of any information, apparatus, product, or process disclosed, or represents that its use would not infringe privately owmed rights. Reterence herein to any specific commercial product, process, or service by trade name, trademark, manufacturer, or othenwise, does not necessarily constitute or imply its endorsement, recommendation, or favoring by the United States Government or any agency thereof. The views and opinions of authors expressed herein do not necessarily state or reflect those of the United States Govemment or any agency thereof.

Superconducting Super Collider Laboratory is an equal opportunity employer. 


\section{DISCLAIMER}

Portions of this document may be illegible in electronic image products. Images are produced from the best available original document. 
SSCL-622

\title{
Is Transverse Feedback Necessary for the SSC Emittance Preservation? (Vibration Noise Analysis and Feedback Parameters Optimization)
}

\author{
V. V. Parkhomchuk, * V. D. Shiltsev* \\ Superconducting Super Collider Laboratory ${ }^{\dagger}$ \\ 2550 Beckleymeade Ave. \\ Dallas, TX 75237
}

June 1993

*Guest Scientist from Budker Institute of Nuclear Physics Novosibirsk, Russia.

†Operated by the Universities Research Association, Inc., for the U.S. Department of Energy under Contract No. DE-AC35-89ER40486. 


\title{
Is Transverse Feedback Necessary for the SSC Emittance Preservation? (Vibration Noise Analysis and Feedback Parameters Optimization)
}

\author{
V. V. Parkhomchuk and V. D. Shiltsev \\ Visitors from Budker Institute for Nuclear Physics, \\ 630090 , Novosibirsk, Russia
}

\begin{abstract}
The paper considers the Superconducting Super Collider (SSC) site ground motion measurements as well as data from accelerators worldwide about noises that worsen beam performance. Unacceptably fast emittance growth due to these noises is predicted for the SSC. A transverse feedback system was found to be the only satisfactory alternative to prevent emittance decay. Optimization of the primary feedback parameters was done.
\end{abstract}




\subsection{INTRODUCTION}

Vibrations of magnetic elements of the Superconducting Super Collider (SSC) can seriously affect proper machine operation. Tolerable SSC vibration levels are at least few orders of magnitude tighter than those for the largest existing hadron accelerators such as the Tevatron and SppS (CERN) because the transverse emittance of the beam will be about 100 times smaller and the ring circumference will be about 10 times larger at the SSC than for these smaller machines. A larger circumference means a lower revolution frequency and lower betatron frequency-dangerous because of the rapid increase of vibrations at lower frequencies. Also, more magnetic elements in the rings can disturb the ideal motion of the beams.

Vibration effects on collider performance have been studied in several works. ${ }^{1-5}$ It was found that depending on the frequency of the noise, one can distinguish two mechanisms of beam perturbation. At low frequencies (much less than the revolution frequency), the noise produces a distortion of the closed orbit of the beam. High frequency noises, especially at frequencies near the fractional part of the betatron oscillation frequency, (700-1000 Hz at the SSC) cause direct transverse emittance growth.

Insufficient information was available about: (1) noises at the most interesting frequencies in the $\mathrm{kHz}$ range, and (2) about powerful vibrations at frequencies below $1 \mathrm{~Hz}$. Such data is needed to decide if any kind of transverse beam motion damper is necessary. Analysis of our recent measurements at the SSC site $^{6}$ and beam motion data from accelerators worldwide (Section 2) indicate the need for a transverse beam-based feedback system to control emittance growth.

Section 3 is devoted to optimization of main parameters of the feedback system (such as gain, frequency band, etc.) needed to minimize emittance growth.

Main conclusions are summarized in Section 4.

\subsection{ANALYSIS OF SOURCES}

\subsection{General Consideration}

The primary sources which lead to emittance growth in large hadron colliders are quadrupoles (quad) jitter and high-frequency variations of the bending magnetic field in dipoles. ${ }^{1,2}$ Both sources produce angular kicks and disturb beam motion. In the simplest case when the kick amplitude $\delta \theta$ varies randomly after the revolution time $1 / f_{0}$ and has dispersion $\delta \theta^{2}$, one can estimate the transverse emittance growth as:

$$
d \varepsilon / d t=1 / 2 f_{0} \delta \theta^{2}\langle\beta\rangle N .
$$

where $\langle\beta\rangle$ is the average beta function, and $N$ is the number of elements which produce kicks. Generally, when external noise is not "white" (exactly random) and can be described by spectral density of power $S_{\delta \theta}(f)$ which depends on frequency $f$, emittance growth calculated in References 1 and 2 is:

$$
d \varepsilon / d t=1 / 2 f_{0}^{2} \sum_{i}\left(\beta_{i} \operatorname{Sum}_{i}(v)\right) .
$$

where $\operatorname{Sum}_{i}(v)=\Sigma S_{\delta \theta}\left(f_{0}(v-n)\right)$ is the sum of power spectral densities of angular kicks produced by the $\mathrm{i}$-th source at a set of frequencies since the fractional part of betatron oscillation frequency $f_{0}|v-n|$, $f_{0}$ is the revolution frequency and $\beta_{i}$ is the beta function at the $\mathrm{i}$-th element. The dimension of $\operatorname{Sum}(f)$ is $1 / \mathrm{Hz}$, so the dimension of the emittance growth rate is meters/sec.

Applying this formula to the SSC gives the following acceptable levels for a 20 -hour doubling time for initial emittance $\varepsilon=4.7 \times 10^{-9} \mathrm{~cm}$, Reference 2 : 
Single quadrupole transverse vibration spectral density of power is limited by the value of

$$
\sum_{n} S_{d}\left(f_{0}(v-n)\right)<3 \times 10^{-12} \mu \mathrm{m}^{2} / \mathrm{Hz}
$$

or the rms amplitude of turn-to-turn jitter of each quadrupole (white noise in frequency band $f_{0}$ ):

$$
d_{\mathrm{rms}}<10^{-4} \mu \mathrm{m},
$$

and a tolerable level of bending magnetic field fluctuations to its mean value $B$ in the dipole: 2

$$
\delta \mathrm{B}_{\mathrm{rms}} / B<7 \times 10^{-10} \text {. }
$$

At frequencies lower than the fractional part of the betatron oscillation frequency, for the SSC $\left(f_{0}(\nu-n)\right)_{\min }=0.28 * 3.4 \mathrm{kHz}=952 \mathrm{~Hz}$, disturbances of the beam lead to closed orbit distortions (COD). If the COD value at the Interaction Point (IP) is more than the beam size $\sigma^{*}$ at the IP, collider luminosity decreases significantly, i.e., it will be similar to increasing effective emittance.

Beam-beam separation can lead to emittance growth due to non-linear beam-beam effects. The acceptable separation level depends on the machine parameters and usually shouldn't exceed $0.1-0.2 \sigma^{*}$, Reference 2 . For the SSC this limitation can accept a COD level in the lattice of $5.5 \mu \mathrm{m}$, and a level of beam-beam separation at the IP of $0.5 \mu \mathrm{m}$.

\subsection{Measurements at the SSC Site and Estimations of Emittance Decay Time}

SSC site vibration measurements 6 allow estimating preliminary beam motion using vibration spectral and correlation characteristics data. Figure 1 taken from Reference 6 shows both the most quiet and rather noisy ground vibration spectra compared with the preceding COD and emittance growth limitations. One can see that the measured high frequency vibration level is only marginally acceptable. At frequencies of about $0.1 \mathrm{~Hz}$ and below, the level of ground motion is significantly high but there is a lack of information about space correlation of such motion. Some experimental data show significant uncorrelated motion of different points of the ground even for very slow processes. ${ }^{7}$

In the high-frequency region above $300 \mathrm{~Hz}$ the spectrum of ground vibrations behaves vs. frequency $f$ as $A / f^{4}$. At the first resonant frequency $f_{\min }=952 \mathrm{~Hz}$ the value of the spectrum is equal to

$$
A / f_{\min }^{4}=3 \times 10^{-14}-6 \times 10^{-11} \mu \mathrm{m}^{2} / \mathrm{Hz},
$$

and depends on conditions ("quiet" or "noisy"). Corresponding to this spectrum value, the "white noiselike" rms amplitude of vibration is $1 \times 10^{-5}-5 \times 10^{-4} \mu \mathrm{m}$. If every quadrupole of the SSC will vibrate as measured on the ground, the estimated emittance doubling time $\tau$ is

$$
\tau=50 \mathrm{~min}-2000 \text { hours. }
$$

Moreover, SSC dipole vibration measurements ${ }^{6}$ have shown that at this frequency region mechanical resonances amplify ground vibrations at a factor of $Q=3-10$, so in the worst case when mechanical and betatron oscillations are in resonance, emittance doubling time falls as

$$
\tau=(50 \mathrm{~min}-2000 \mathrm{hrs}) / Q^{2}=30 \mathrm{sec}-20 \text { hours. }
$$

These figures cause concern. Additional detailed measurements under more realistic conditions are needed. Considerations include underground tunnel vibrations, all accelerator accessories working (compressors, power supplies, etc.), mechanical resonances of connected magnet strings, real magnet supports, etc. Some imagination on creating a "working machine environment" is necessary. In the next section we examine the usual accelerator vibroclimate from existing accelerator data. 


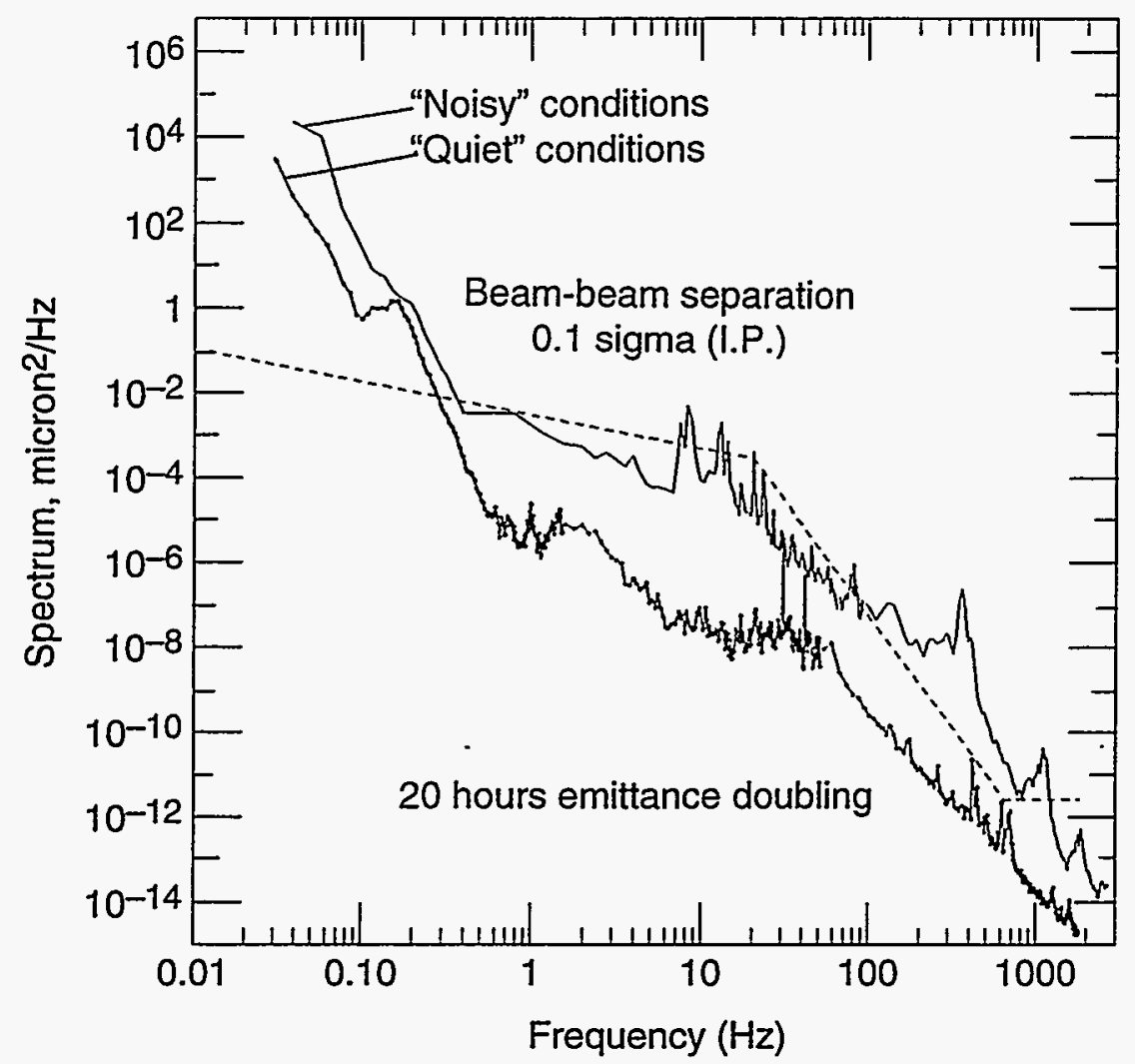

Figure 1. Spectral Density of Power of Vibrations in "Quiet" and "Noisy" Conditions (Solid Curves), Reference 6. Dashed lines indicate the level of vibration which cause $0.1 \sigma \mathrm{IP}$ beam-beam separation and the SSC emittance doubling after 20 hours of collider operation.

\subsection{Analysis of Existing Accelerator Operation}

As previously noted, the emittance condition depends strongly on the accelerator condition (environment, mechanics, etc.). To use beam observation data from different machines to understand nominal "accelerator conditions," a common view point is needed. Accelerators differ in many parameters (energy, revolution frequency, tune, etc.) and direct application of data from one machine to another is difficult.

We suggest the following considerations. Assume that the beam moves due to independent motion of quads only. Assume the absence of correlation for two different quads motions is reasonable at a wide frequency range (References 6 and 8). Next, find what value of single quad vibration $\delta x$ should cause the observed beam motion characteristics.

Low frequency (in comparison with the revolution frequency) movements of quads leads to COD, as noted previously. One can assume that motions of different lenses are uncorrelated (measurements show that the assumption isn't fully valid only at frequencies below units of $\mathrm{Hz}$ ). Then the rms value $\Delta X$ of COD in the point with beta function $\beta$ can be estimated as in Reference 1:

$$
\Delta X^{2}=\delta x^{2} /\left(8 \sin ^{2}(\dot{\pi} v)\right) \underset{i}{\Sigma}\left(\beta_{i} / F_{i}^{2}\right)
$$


where $v$ is the tune, the sum $\Sigma$ means the summation over all quads, $\beta_{i}$ and $F_{i}$ are the beta function in the $i$-th quad and the focal length of the quad, respectively. For a FODO periodic lattice (used at many machines) the sum is equal to:

$$
\sum_{i} \beta_{i} / F_{i}^{2}=4 N \operatorname{tg}(\mu / 2) / L
$$

where $N$ is full number of quads, $L$ and $\mu$ are the distance between quads and the phase advance per FODO cell, respectively. Taking into account that $N L \simeq 2 \pi \beta v, \beta$ is mean beta function, one can transfer Eq. (9) into:

$$
\Delta X^{2}=\delta x^{2} 4 \pi v / \sin ^{2}(\pi v)\left[(\beta / 2 L)^{2} \operatorname{tg}(\mu / 2)\right]
$$

For Alternating Gradient (AG) accelerators the factor in the square brackets is about 1 , so one can estimate single-quad amplitude $\delta x$ with an accuracy of about $20 \%$ :

$$
\delta x^{2}=\Delta X^{2} \quad \sin ^{2}(\pi v) /(4 \pi v),
$$

or, in terms of spectral power density $S(f)$ :

$$
S(f)=\delta x^{2} / f=\Delta X^{2} \sin ^{2}(\pi v) /(4 \pi v f) .
$$

Equation 13 will be applied to the spectrum of vertical motion of the beam orbit in the electron ring of HERA, ${ }^{9}$ to the spectrum of COD in the Novosibirsk VEPP-3 electron storage ring, ${ }^{10}$ and to slow orbit drifts data, Table 1.

TABLE 1. ORBIT DRIFTS DATA.

\begin{tabular}{|lcccccc|}
\hline & $\Delta x, \mu \mathrm{m}$ & $\tau$ & $v$ & $t, \mathrm{~Hz}$ & $S, \mu \mathrm{m}^{2} / \mathrm{Hz}$ & Ref. \\
\hline KEK Photon Factory COD & 100 & $2 \mathrm{hrs}$ & 3.3 & $2 \times 10^{-5}$ & $7 \times 10^{6}$ & 11 \\
HERA (e) & 200 & $80 \mathrm{sec}$ & 45 & $2 \times 10^{-3}$ & $1.5 \times 10^{4}$ & 9 \\
\hline
\end{tabular}

At high frequencies one can estimate equivalent quadrupole vibration level $\delta x^{2}$ from the rate of emittance growth, see Eq. (1) and take into account the FODO lattice Eq. (10):

$$
d \varepsilon / d t=2 f_{0} N \operatorname{tg}(\mu / 2) \delta x^{2} / L \simeq 32 / \pi^{2} c \delta x^{2}(v / R)^{2}\left[\operatorname{tg}(\mu / 2) /(2 \mu / \pi)^{2}\right],
$$

where $c$ - the velocity of light and $R$ - average radius of the accelerator. For AG machines the factor in square brackets is about 1 , so:

$$
\delta x^{2}=d \varepsilon / d t R^{2} /\left(3 c v^{2}\right)
$$

Because the emittance growth is determined by the spectral density of power at the fractional part of betatron oscillation frequency $f_{\min }$, then one can estimate the spectrum of quad vibrations $S\left(f_{\min }\right)$ :

$$
S\left(f_{\min }\right) \simeq \delta x^{2} / f_{\min }=d \varepsilon / d t R^{2} /\left(3 c v^{2} f_{\min }\right) \text {. }
$$


Equation (19) will be applied to several emittance growth data, Table 2.

TABLE 2. EMITTANCE GROWTH DATA.

\begin{tabular}{|llllll|}
\hline & $\gamma d \varepsilon / d t, \mu \mathrm{m} / \mathrm{hr}$ & $v$ & $f_{0} \Delta V, \mathrm{kHz}$ & $S, \mu \mathrm{m}^{2} / \mathrm{Hz}$ & Ref. \\
\hline Tevatron & 10 & 19.05 & 2.5 & $3.3 \times 10^{-9}$ & 12 \\
Tevatron & 0.43 & 19.41 & 20 & $2.0 \times 10^{-11}$ & 13 \\
HERA $(p)$ & 1.0 & 31.29 & 13 & $6.0 \times 10^{-11}$ & 14 \\
\hline
\end{tabular}

The preceding data together with the results of SSC site measurements ${ }^{6}$ are shown in Figure 2 . The dashed line in Figure 3 presents a rough fit according to the formula:

$$
S(f)=0.03 / f^{2} \quad\left[\mu \mathrm{m}^{2} / \mathrm{Hz}\right] \text {. }
$$

Figure 2 shows that beam-based data and ground vibration measurements made under noisy conditions differ slightly from each other in the frequency range $0.5-50 \mathrm{~Hz}$.

The difference at low frequencies (ground vibration greater than COD) is connected with high correlation of different quads motion at low frequencies. For true calculation of COD one should take into account uncorrelated motion of quads. For large accelerators a "Fractal Model of Ground Motion" works well at low frequencies. ${ }^{7}$ According to Reference 7 relative (uncorrelated) displacement $\Delta x$ of two points distanced by $l$ after the time interval $\tau$ can be estimated by the empirical formula ("ATL-law"):

$$
\Delta x^{2}=A \tau l, A \simeq 10^{-4} \mu m^{2} /(\text { meter } \cdot \sec ),
$$

then estimation of $\mathrm{COD}^{1}$

$$
\left\langle\Delta X^{2}\right\rangle \simeq(A C \tau \beta) /\left(4 \sin ^{2}(\pi \nu)\langle\beta\rangle\right)
$$

where $\langle\beta\rangle$ and $\beta$ are mean and point of observance beta functions, $C$ is the machine circumference.

The corresponding spectral density of power should be estimated as:

$$
S(f) \simeq \delta x^{2} / f \simeq A L / f^{2} .
$$

It is difficult to predict the value of the site dependent constant $A$, but if one takes $A=10^{-4} \mu \mathrm{m}^{2} / \mathrm{Hz}$ from UNK measurements ${ }^{15}$ and $L \simeq 100 \mathrm{~m}$ then

$$
S(f)=0.01 / f^{2}
$$

which is very close to the Figure 2 fitted curve in amplitude and has the same frequency dependence, see Eq. (17).

Another difference between beam and ground motion data (see Figure 2) is at frequencies above a few hundred Hz. It seems that ground motion is overlaid by a stronger effect. The next section will give a possible explanation of this effect. 


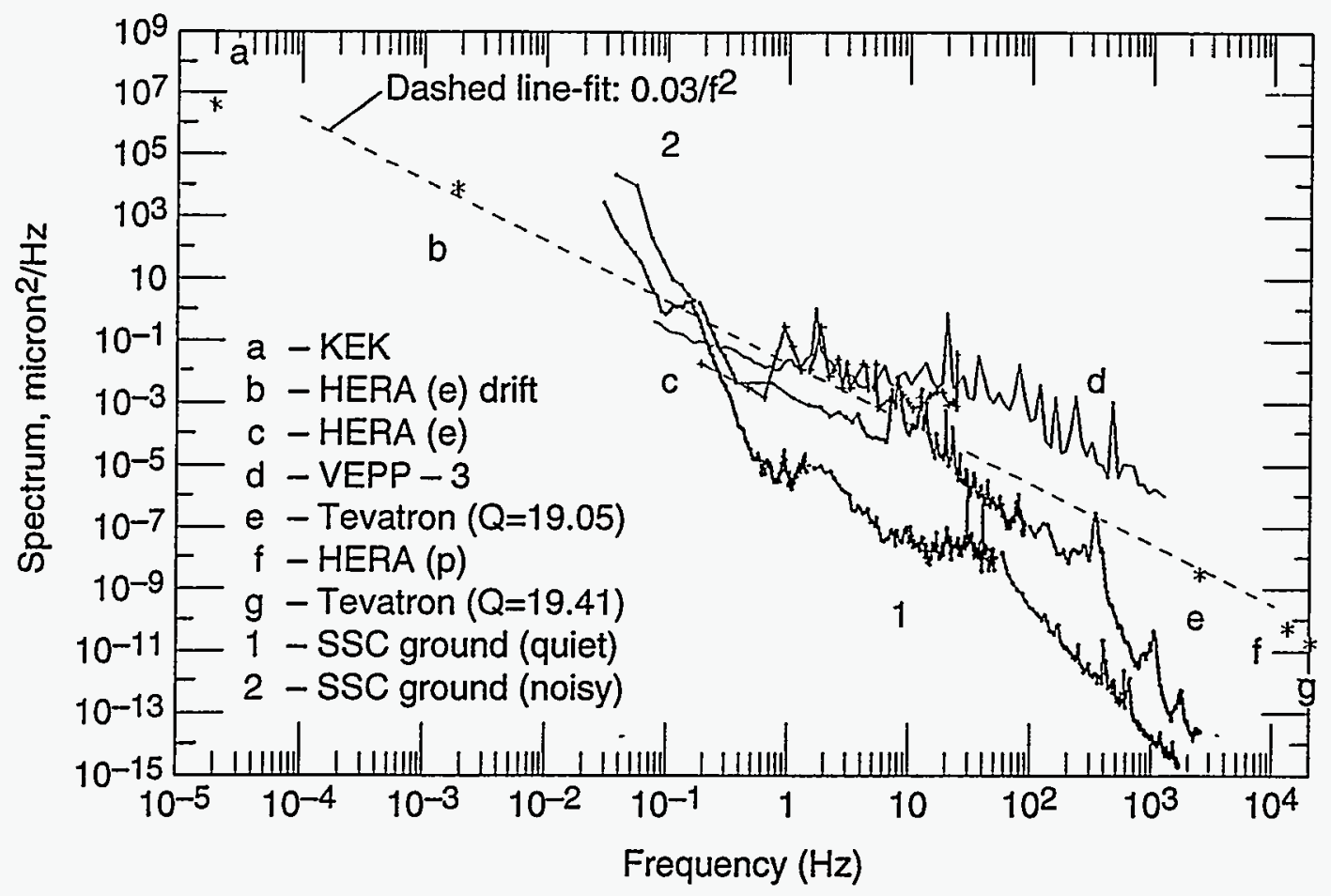

Figure 2. Spectra of Vibrations at the SSC Site and Worldwide Accelerator Quads Vibrations Calculated from Beam Data (See Comments in the Text).

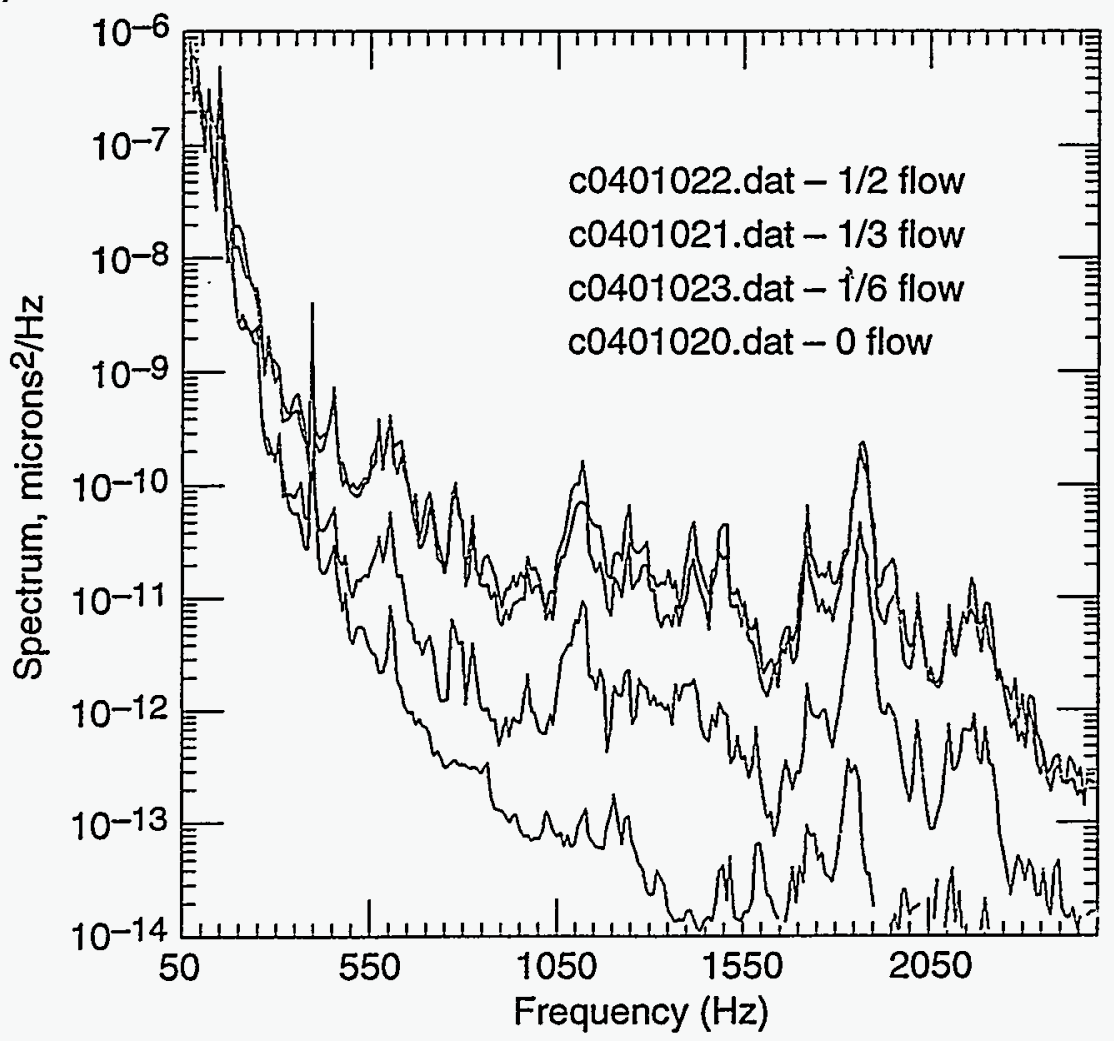

Figure 3. Spectra of Vibrations of Pipe with Different Air Flow Rates (See Comments in the Text). 


\section{4 "New" Effect - Turbulent Liquid or/and Gas Flow}

The Figure 2 non-coincidence may be caused by turbulent flow-induced vibrations in cooling pipes and coils of magnetic elements. In normal conductive magnets it's cooling water. In the case of the SSC and HERA - the accelerators with superconducting magnetic elements-flows of liquid and gas helium, and nitrogen will generate beam pipe vibrations. At frequencies of about $1 \mathrm{kHz}$, skin effect leads to a corresponding jitter of the magnetic axis in quadrupoles because the field is "frozen" inside the pipe.

The "frozen field" effect in dipoles produces a bending field variation if turbulent flow generates vibration affecting beam pipe shape, such as quadrupole oscillation of the pipe cross section. The tolerable level $d x$ for amplitude of such vibration can be estimated through the level of magnetic field fluctuation $\delta B / B$ (Eq. (5)):

$$
d x \simeq \delta B / B \cdot b
$$

where $b$ is radius of beam pipe. For the SSC $b \simeq 2 \mathrm{~cm}$ and $d x$ is about $1.4 \times 10^{-9} \mathrm{~cm}$. This is about 7 times less than the restriction on a single-quadrupole vibration (see above).

The experimental data indicate the danger of flow turbulence. The expected liquid He flow rate for the SSC dipole is about $100 \mathrm{~g} / \mathrm{sec}$. Figure 3 shows the spectra of vibration of a 5-m long, 5-cm diameter pipe with a normal air flow rate of about $0.02 \mathrm{~g} / \mathrm{sec}$. Figure 4 (taken from Reference 15) shows the spectra of vibration of a $1-\mathrm{m}$ long, $2.5-\mathrm{cm}$ diameter pipe with water flow rate up to $18 \mathrm{~g} / \mathrm{sec}$. In comparison with ground motion these spectra are 2-5 orders higher (i.e., 10-300 times greater in amplitude).

Another interesting feature of spectra presented in Figures 3 and 4 is the slope of the curves. At frequencies above a few hundred $\mathrm{Hz}$, these spectra fall with frequencies of $f^{-(1.0 \div 2.5)}$. These spectra differ from ground motion law $f^{-(3.5+4.5)}$ and more closely follow data observed at several accelerators (see Figure 2).

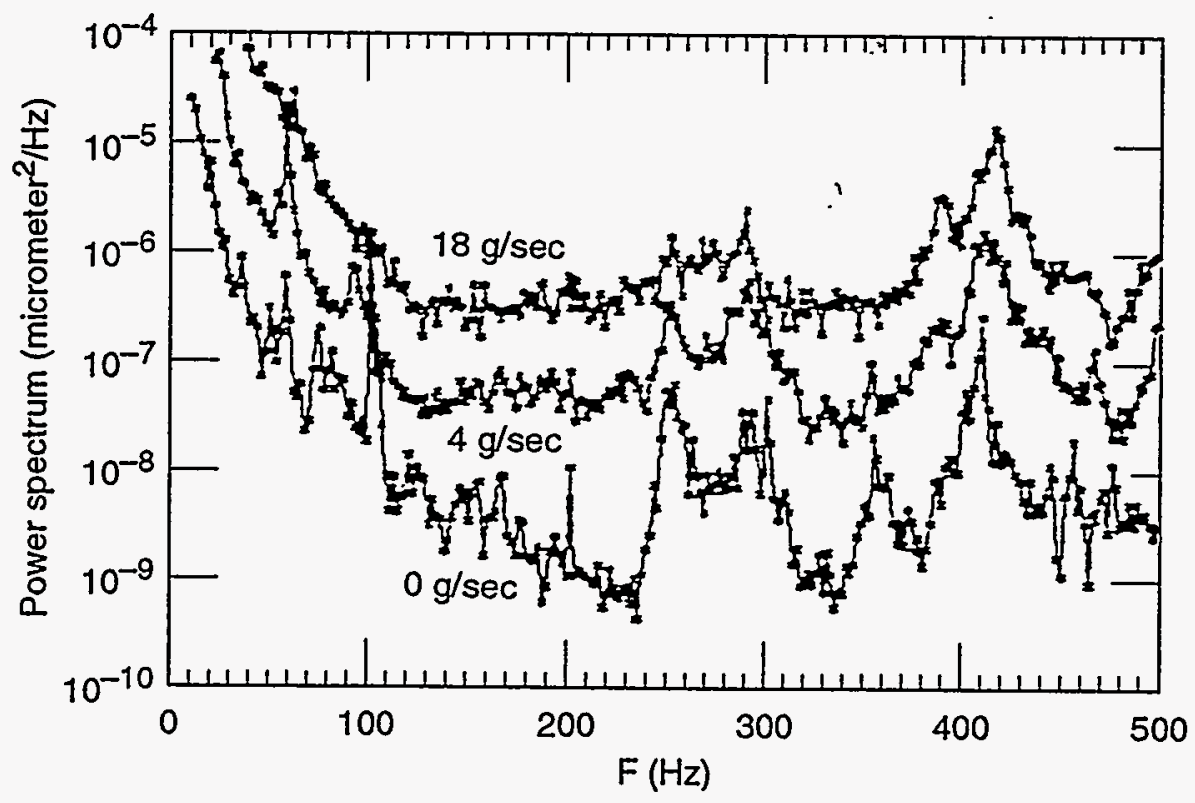

Figure 4. Spectra of Vibrations of a Pipe with Different Water Flow Rates (See Comments in the Text). 


\subsection{A Way for Solving the Problem}

There are two ways to control noise influence on the SSC beam: the first one is mechanical improvement and decreasing the man-made vibrations level; the second is use of a beam-based transverse feedback system. Both ways should be used.

Mechanical improvements (such as vibro-isolation and damping systems) have some drawbacks:

1. Mechanical methods cannot prevent excitation of vibrations born inside the magnet;

2. Every magnetic element (or most of them) should have improved support; however, such supports are very costly because of the complexity of the special mechanics;

3. A tradeoff of such supports is that damping vibrations at one set of frequencies may result in the amplification of vibrations at another.

4. It is very difficult to stabilize mechanically (by passive devices) low-frequency magnet motion (with periods greater than $10 \mathrm{sec}$ ).

A feedback system directly influences the beams, so the primary drawback is the possibility of "heating" the beam additionally by the properly applied system noises. However, only one (or a few) feedback loop is necessary for the whole collider. Therefore, distributed improvement attempts are not needed. The right choice of feedback system parameters can prevent the preceding beam-disturbing effects. The following section discusses SSC feedback system optimization.

\subsection{FEEDBACK OPTIMIZATION}

\subsection{General Considerations}

Ordinarily, any feedback system can be presented as the superposition of a part which describes the beam motion without the system (open loop) and a proper feedback (see Figure 5). Because both beam motion and feedback generally are frequency dependent, the first part is characterized by beam response function $G(\omega)$ on an external excitation without feedback and the second one by feedback gain function $H(\omega)$. Both functions $G(\omega)$ and $H(\omega)$ are complex. The primary function properties are described and discussed below as follows.

Let's calculate coherent beam motion $X_{\omega b}$ under feedback with noise $X_{\omega p}$ and when external excitation is equal to $X_{\omega 0}$. A simple relation can be written from Figure 5 as follows:

$$
X_{\omega b}=G(\omega)\left(X_{\omega 0}-H(\omega)\left(X_{\omega b}+X_{\omega p}\right)\right)
$$

Then one can find the solution as:

$$
X_{\omega b}=\left[G(\omega) X_{\omega 0}-H(\omega) G(\omega) X_{\omega p}\right] /[1+G(\omega) H(\omega)],
$$
or for spectral power of beam coherent motion $S_{b}(\omega) d \omega=\left\langle\left|X_{\omega b}\right|^{2}\right\rangle$ (brackets indicate averaging over
time):

$$
S_{b}(\omega)=\left[|G(\omega)|^{2} S_{0}(\omega)+|H(\omega) G(\omega)|^{2} S_{p}(\omega)\right] /|1+G(\omega) H(\omega)|^{2}
$$


where $S_{0}(\omega)$ and $S_{p}(\omega)$ are power spectral density of external noises and noises of feedback system, respectively.

Here we took into account that noises of feedback are independent from external noises $\left\langle X_{\omega 0} X^{*}{ }_{\omega p}\right\rangle=0$. For stationary random processes such as all noises, the unit of spectral power density $S(\omega)$ is micrometers ${ }^{2}$ seconds. The rms value of beam coherent motion is equal to the square root of integral of $S_{b}(\omega)$ over the frequency band $d \omega$.

Coherent beam motion leads to emittance growth due to decoherence (which is included in the $G(\omega)$ function). We'll try to minimize coherent beam motion by using properties of functions $S_{p}(\omega), S_{0}(\omega)$, $G(\omega)$, and $H(\omega)$.

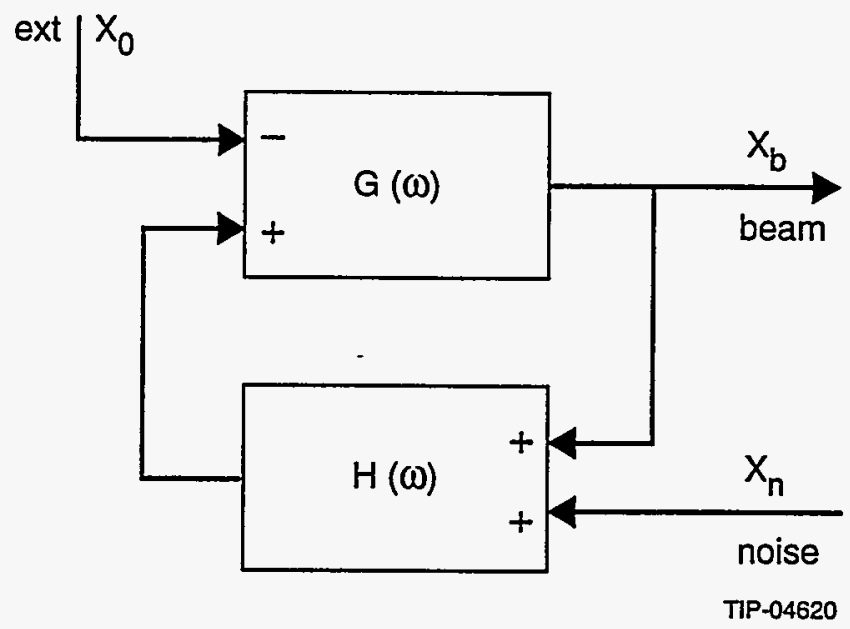

Figure 5. General Scheme of a Beam-based Feedback.

\subsection{Estimation of Beam Response Function G ( $\omega)$}

Let's calculate the beam response with external excitation by a periodic force with frequency $\omega$ which produces angular kick $a_{0} \exp (i \omega n T)$ at $\mathrm{n}$-th tum ( $T$ is the revolution period). Working in normalized units of beam coordinate $x$ and velocity $v$ (in which emittance is equal to $x^{2}+v^{2}$ ) one can link the variables $x^{n+1}$ and $v^{n+1}$ at $(\mathrm{n}+1)$-th turn with $\mathrm{n}$-th turn coordinates $x^{n}$ and $v^{n+1}$ according to the following equations:

$$
\begin{aligned}
& x^{n+1}=D\left[x^{n} \cos (\mu)+v^{n} \sin (\mu)\right] \\
& v^{n+1}=D\left[v^{n} \cos (\mu)-x^{n} \sin (\mu)\right]+a_{0} \exp (i \omega(n+1) T)
\end{aligned}
$$

where factor $D$ describes a slight coherent oscillation amplitude decrease caused by the decoherence process during a one-turn revolution (we estimate this factor in terms of the number of turns $N_{d}$ necessary for $1 / e$ times damping of coherent motion as $\left.D \simeq 1-1 / N_{d}\right), \mu$ is the betatron phase advance over one turn. The solution of Eq. (26) which describes forced oscillations is $(x, v)^{n}=\left(x_{0}, v_{0}\right) \exp (i \omega n T)$, where :

$$
x_{0} / a_{0}=G(\omega)=\frac{D \sin (\mu)}{\exp (i \omega T)+D^{2} \exp (-i \omega T)-2 D \cos (\mu)}
$$


At low frequencies when $\omega T<<1$, the response function describes the amplitude of closed orbit distortion:

$$
G(\omega \rightarrow 0)=\frac{\sin (\mu)}{2-2 \cos (\mu)}=\frac{1}{2 \operatorname{tg}(\mu / 2)}
$$

At frequencies close to betatron resonances when $\omega T=2 \pi n \pm \mu+\Delta \omega$ ( $n$ is integer), the response function is equal to:

$$
G(\Delta \omega)=\frac{ \pm i}{2(1-D+i \Delta \omega T)}=\frac{ \pm i N_{d}}{2\left(1+i \Delta \omega T N_{d}\right)} .
$$

Exactly at resonances $\omega_{n} T=2 \pi n \pm \mu$ and according to Eq. (29) the value of the response function is $\left|G\left(\omega_{n}\right)\right|=N_{d} / 2$ and the width of resonances is $\delta \omega=1 /\left(T N_{d}\right)$.

\subsection{Simple Example: RC-Type Feedback without Noises}

To understand the properties of excited coherent oscillations, consider when the feedback has no noises $\left(S_{p}(\omega)=0\right)$ and the feedback amplification function $H(\omega)$ has an integrating type (similar to RC) filter:

$$
H(\omega)=\frac{i H_{\nu}}{1+i \omega \tau}
$$

where $\tau$ is the integration time. At low frequencies $H(0)=i H_{v}$ feedback opposes the transverse velocity of the beam. The beam response to an external excitation in the presence of such feedback will be characterized by response function $R(\omega)=X_{\omega b} / X_{\omega 0}$ :

$$
R(\omega)=G(\omega) /[1+G(\omega) H(\omega)] .
$$

The function $R(\omega)$ will also have resonant properties at frequencies $\omega_{n}$ and one can estimate function behavior as:

$$
|R(\Delta \omega)|=\frac{|G(\Delta \omega)|}{\left|1+G(\Delta \omega) H_{n}\right|} \simeq \frac{N_{d}}{\left|1+N_{d} H_{n} / 2+i\left(\Delta \omega T N_{d}\right)\right|},
$$

where $H_{n}=H\left(\omega_{n}\right)$ are the feedback amplification values at the resonant frequencies.

If the feedback is powerful $\left|H_{n}\right|>1 / N_{d}$ when compared to the case when $H=0$, the response function values at resonance will be decreased from $N_{d}$ to $1 / H_{n}$ and the bandwidth will be increased to

$$
\delta \omega=\left(1 / N_{d}+\left|H_{n}\right| / 2\right) / T .
$$

Figure 6 shows an example of calculating the function $R(\omega)$ for integrating a feedback loop, Eq. (30), with parameters $D=1-1 / 500=0.998$ and $\tau=0.0002 \mathrm{~s}$. Solid lines correspond to the real part of $R(\omega)$ and dashed lines correspond to the imaginary part of $R(\omega)$. The frequency scale around the betatron harmonics was expanded for better presentation (the widths of resonances are very small in the usual scale). 
In Figure 6 the stars $*$ show the positions of the poles of complex function $R(\omega)$ (i.e., complex frequencies $\omega^{\prime}$ for which $1 / R(\omega)=0$ ):

$$
\operatorname{Re}\left(\omega^{\prime}\right) \simeq \omega_{n} ; \operatorname{Im} \omega^{\prime}=\frac{2}{\frac{d}{d \omega}[\operatorname{Re}(R(\omega)) / \operatorname{Im}(R(\omega))]}
$$

For simul taneous with $\operatorname{Re}\left(\omega^{\prime}\right)$ presentation $\operatorname{Im}\left(\omega^{\prime}\right)$ was multiplied by factor $[1 /(1-D)] / 2 \pi=N_{d} / 2 \pi$. For example, the ordinate of poles at $H_{\nu}=0$ is equal to $N_{d} / 2 \pi\left(1 / T N_{d}\right)^{\prime}=1 /(2 \pi T)=3440 \mathrm{~Hz} / 2 \pi$ $\simeq 540$ (see Figure 6a).

The position of a pole relative to zero axes determines damping or growth for coherent oscillations at different resonances. A negative imaginary part indicates damping of coherent oscillations; a positive one means that coherent oscillations at this frequency are unstable.

One can see in Figure 6 a, b, c, and d and that with a simple feedback, like Eq. (30), one can strongly damp oscillations on the first betatron mode (with the lowest frequency $\omega_{n}$ ) and simultaneously decrease damping of all next modes. When the feedback strength becomes equal to $\mathrm{H}_{v}=-0.04$, coherent oscillations on the second betatron frequency become unstable (the threshold value depends on parameters $N_{d}$ and $\tau$ ).

It is interesting that the integrating feedback of Eq. (30) is useful for compensating beam interaction with the resistive wall. For resistive wall instability the distribution of decrements over modes looks very similar. Figure $7 \mathrm{~b}, \mathrm{c}$, and d shows how the initial beam response function (Figure 7a) changes when interaction with the wall produces instability. According to Reference 16 the beam-wall interaction can be described as an additional positive feedback response:

$$
H(\omega)=\frac{i H_{v}}{1+i \omega \tau}+\frac{(1+i) \omega \cdot \tau_{w}}{2 N_{w}\left(1+\left(\omega \cdot \tau_{w}\right)^{2}\right)}
$$

where the first term is from Eq. (30) and the second from Reference 16. In calculations shown in Figure 7, values of parameters $\tau_{w}=0.003 \mathrm{sec}$ and $N_{w}=20$ were used -the same as for the SSC collider at $2 \mathrm{TeV}$. Figure $7 \mathrm{~b}$ shows that without feedback $\left(\mathrm{H}_{v}=0\right)$ the first mode with the lowest frequency is unstable (its pole places over the zero axis) and all others are stable. In the case of optimal external feedback with $\mathrm{H}_{v}=-0.12$ and integrating time $\tau=0.0001 \mathrm{sec}$, one can fully to compensate instability (see Figure $7 \mathrm{c}$-all poles have a negative imaginary frequency part). The second mode becomes unstable if the feedback strength is increased to $\mathrm{H}_{v}=-0.05$ (see Figure 7d).

The proper choice of feedback gain (which produces fully stable and maximal beam damping) depends on the spectra of beam noise, the noise of the Beam Position Monitor (BPM), and the coherent interaction of the beam. 

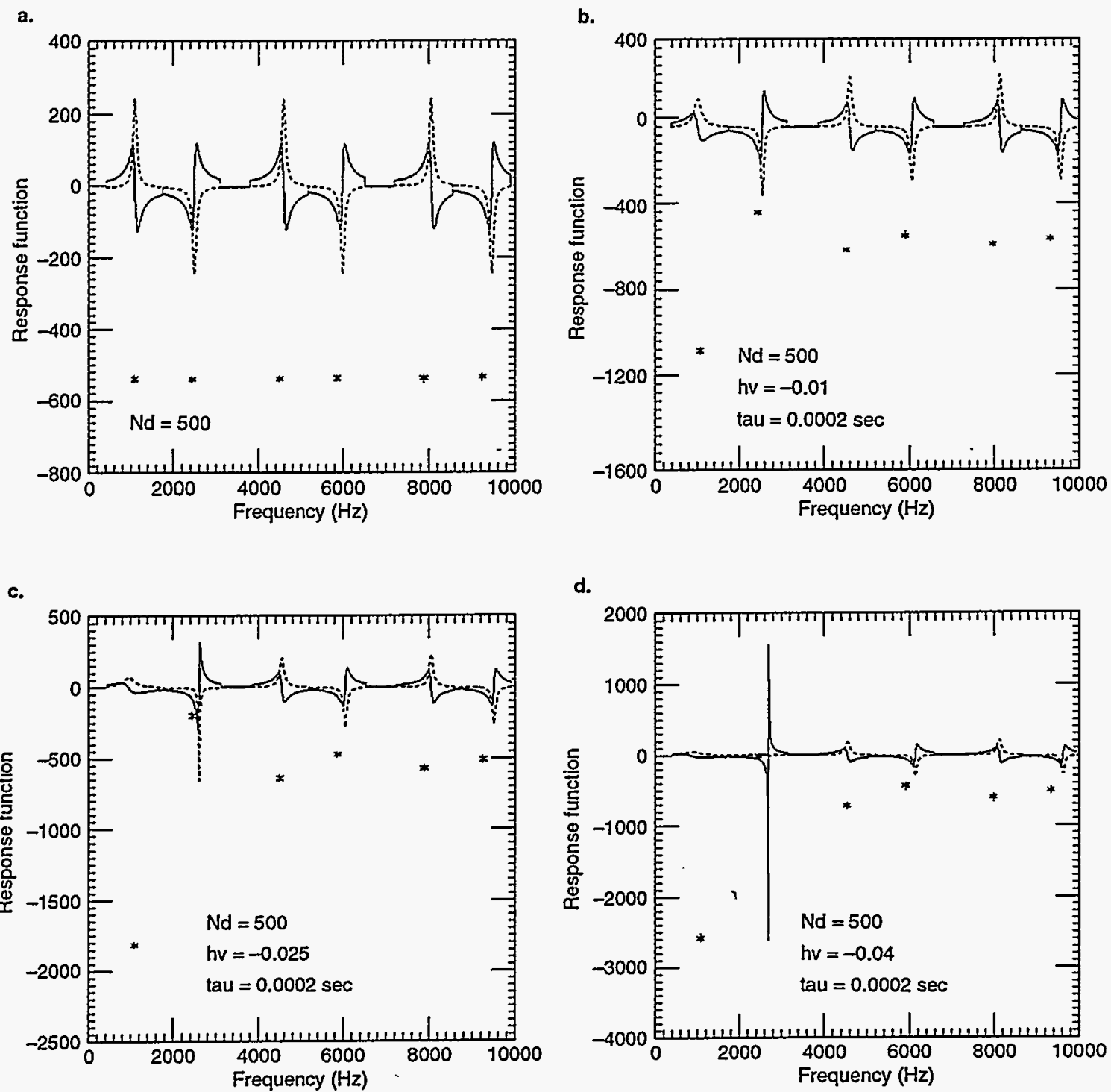

Figure 6. Response Function on External Excitation $R(\omega)$ vs. Frequency for Different Feedback Gain: a) $H_{V}=0$; b) $H_{V}=-0.01$; c) $H_{V}=-0.025$; d) $H_{V}=-0.04$. Integrating feedback with time of integration 0.0001 sec. Positions of poles of the function $R(\omega)$ are marked by stars. 

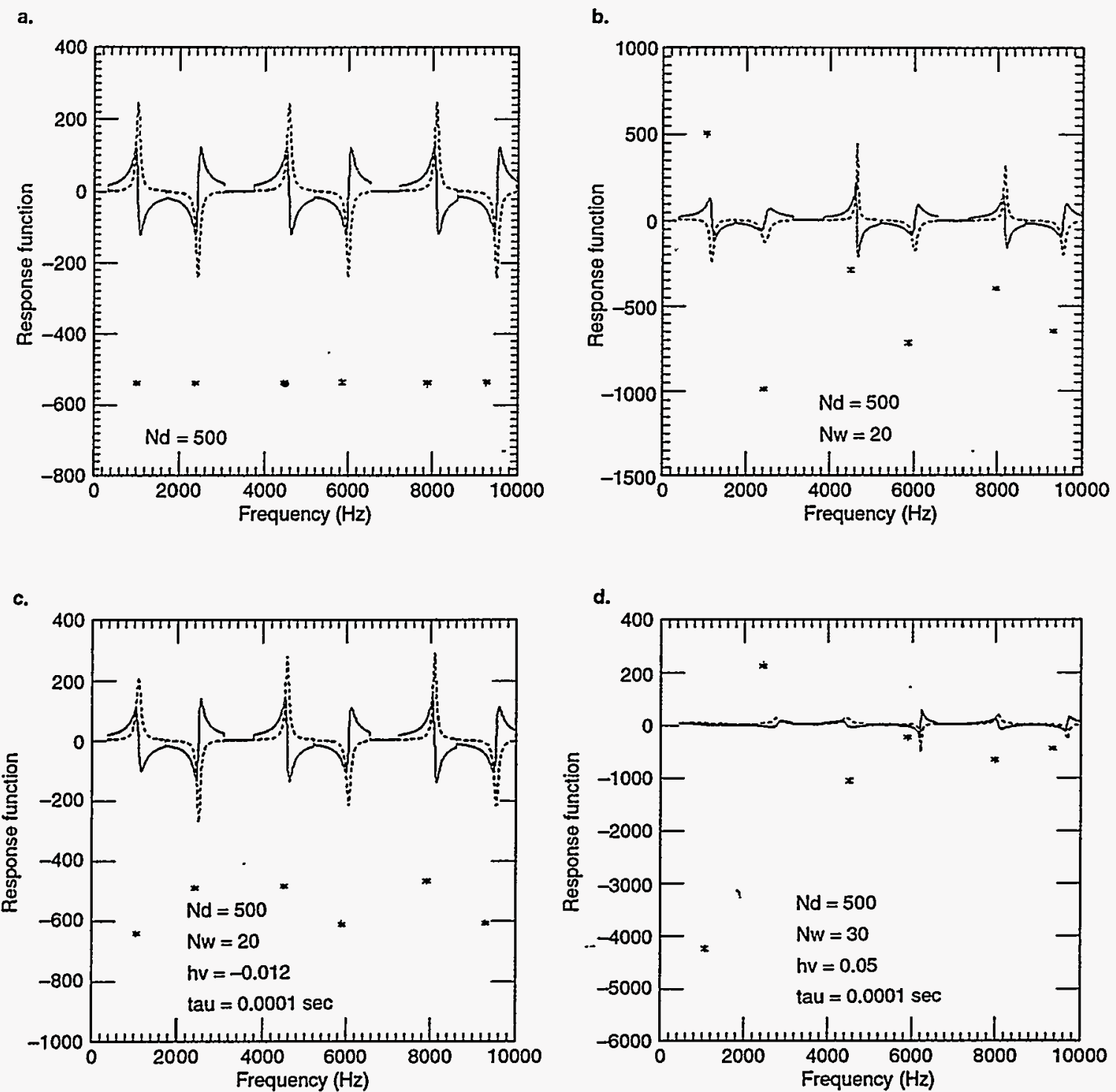

Figure 7. Response Function on External Excitation $R(\omega)$ vs. Frequency with and without Resistive Wall Impedance and with Different Feedback Gain: a) $H_{v}=0$, no resistive wall; b) $H_{v}=0$; resistive wall with $N_{W}=20$; c) $H_{V}=-0.012 ; N_{W}=20$; d) $H_{V}=-0.05, N_{W}=30$. Integrating feedback with time of integration 0.0001 sec. Positions of poles of the function $R(\omega)$ are marked by stars. 


\subsection{Noises of Feedback}

The spectral density of BPM noise $U^{2}(\omega)$ comes from pickup preamplifier input resistor noise which is estimated as follows:

$$
U^{2}(\omega)=\frac{2}{\pi} k T Z * A\left(N B: \text { units Volt }{ }^{2} \mathrm{sec}\right)
$$

where $k$ is Boltzmann's constant, $T$ is the temperature of the resistor, $Z$ is the impedance of the pickup, and $A$ is the amplification factor which depends on the preamplifier construction (usually $A=1-10$ ). If the beam current is equal to $I$, then one can estimate the BPM noise for pickups with aperture $b$ as:

$$
S_{p}(\omega)=\frac{2 k T A}{\pi^{2} Z} b^{2} .
$$

Let's take parameters $I=50 \mathrm{~mA}, T=300 \mathrm{~K}, Z=50 \mathrm{Ohms}, A=10, b=3 \mathrm{~cm}$, and then $S_{p}(\omega)=$ $2 \times 10^{-10} \mu \mathrm{m}^{2} \mathrm{~s}$. To measure the motion of every bunch during the collider operation, the frequency band should be about $\Delta f=60 \mathrm{MHz}$ and the rms amplitude of pickup noise will be $\delta X=$ $\left(S_{p}(\omega) 2 \pi \Delta f\right)^{0.5}=0.26 \mu \mathrm{m}$. However, if we measure the position of the beam by pickup with a frequency band near the revolution frequency (full-turn integration) then the rms amplitude of pickup noise will be equal to $0.001 \mu \mathrm{m}$.

\subsection{Optimization of Feedback}

The emittance growth rate $d \varepsilon / d t$ in large hadron colliders in the presence of a transverse feedback system is determined by coherent beam motion at resonant frequencies ${ }^{1,2}$ (excited by external noise and noise of the feedback); therefore, one can present the rate summed over all modes as:

$$
\frac{d \varepsilon}{d t}=\sum_{n} \frac{\varepsilon_{c n} f_{0}}{1+G\left(\omega_{n}\right) H\left(\omega_{n}\right) \mid N_{d}}
$$

where index $n$ means $\mathrm{n}$-th resonant frequency, see Eq. (29), $\varepsilon_{c n}=A_{n}^{2} / \beta$ is the coherent emittance, $A_{n}$ is the amplitude of coherent betatron oscillations at $n$-th mode, $\beta$ is the value of the beta function, and $f_{0}=1 / T$ is the revolution frequency.

One can estimate the mean-squared amplitude of coherent motion. $A_{n}^{2}$ at the n-th mode as the integral of $S_{b}(\omega)$ over the frequency band $\delta \omega$, Eq. (33):

$$
A_{n}^{2}=\int S_{b}(\omega) d \omega \simeq S_{b}\left(\omega_{n}\right) \delta \omega=f_{0} N_{d}\left[\frac{S_{0}\left(\omega_{n}\right)}{\left|1+N_{d} H_{n}\right|}+\frac{\left|H_{n}\right|^{2} S_{p}\left(\omega_{n}\right)}{\left|1+N_{d} H_{n}\right|}\right]
$$

The corresponding growth of beam size $\left\langle x^{2}\right\rangle$ in the kicker position can be calculated from Eqs. (38) and (39):

$$
\frac{d\left\langle x^{2}\right\rangle}{d t}=\beta \frac{d \varepsilon}{d t}=f_{0}\left[\frac{\Delta X_{0_{n}}^{2}}{\left|1+N_{d} H_{n}\right|^{2}}+\frac{\left|H_{n}\right|^{2} \Delta X_{p_{n}}^{2}}{\left|1+N_{d} H_{n}\right|^{2}}\right]
$$




$$
\begin{aligned}
& \Delta X_{o n}=\left(S_{0}\left(\omega_{n}\right) / T\right)^{0.5} \text { is an amplitude of random single-turn disturbance due to external noise } \\
& \Delta X_{p n}=\left(S p\left(\omega_{n}\right) / T\right)^{0.5} \text { is an amplitude of the noise pickup signal. }
\end{aligned}
$$

The procedure of minimizing $A_{n}$ vs. gain of feedback $H_{n}$ gives the optimum gain for the $n$-th mode:

$$
\left|H_{n}\right|=\sqrt{S_{0}\left(\omega_{n}\right) / S_{p}\left(\omega_{n}\right)}=\Delta X_{o n} / \Delta X_{p n}
$$

One can see that in the case when the spectrum of external noises falls rapidly with increasing frequency (as in reality), then the optimal gain should also fall vs. frequency. For a simple numerical example, the spectral density of noise around the first resonance frequency ( $960 \mathrm{~Hz}$ for the SSC with tune $v=123.28$ ) is $10^{-9} \mu \mathrm{m}^{2} / \mathrm{Hz}$ and the spectrum of pickup noise is $1.2 \times 10^{-9} \mu \mathrm{m}^{2 /} \mathrm{Hz}$; then according to Eq. (41) one must choose a feedback gain of about 1 at this frequency. Because the spectrum of external noise falls, then the gain at next harmonics should be significantly reduced. Otherwise, if one uses feedback with the same strong gain for all frequencies up to the collision frequency (wideband bunch-tobunch feedback) then it'll lead to artificially increasing beam heating because of increased feedback noises-the second term in Eqs. (39) and (40) becomes $n \simeq 10^{4}$ times greater (number of bunches in the SSC $\simeq 20000$ ).

The minimum amplitude of coherent oscillations at the n-th mode occurs with the optimal gain, Eq. (41) and is equal to:

$$
A_{n \min }^{2}=2 \Delta X_{0 n} \Delta X_{p n} .
$$

This equation says that if one has a higher level of external noises in the collider then to keep the same level of beam coherent motion, one needs a less noisy pickup (or feedback system in general). Conversely, when the external excitation level is small, one need not be concerned about pickup noises because of the small feedback gain needed.

Estimates with the SSC parameters include: number of turns before decoherence, ${ }^{1,2} N_{d} \cong 1 /(0.2 \xi)$ $\simeq 1300$ (here $\xi=0.0038$ is the parameter of beam-beam interaction); corresponding time of decoherence is equal to $\tau_{d}=N_{d} / f_{0} \simeq 0.38 \mathrm{sec}$; the SSC beam emittance is equal to $\varepsilon_{0}=4.7 \times 10^{-11} \mathrm{~m} * \mathrm{rad}$, so at the point of orbit where the beta function is about $\beta=200 \mathrm{~m}$, the nominal beam size is $\Delta X_{b}=100 \mu \mathrm{m}$.

Without feedback, $\left(H_{n}=0\right)$, at each turn the mean square beam size will be grow to about $\Delta X_{s}=\Sigma \Delta X_{o n}^{2}$. Let's consider that the acceptable lifetime of the SSC luminosity is equal to $\tau_{1}=24$ hours, then the dangerous level of single-turn excitation can be estimated as:

$$
\Delta X_{s}=\Delta X_{b} /\left(f_{0} \tau_{1}\right)^{0.5} \simeq 5 \times 10^{-3} \mu \mathrm{m} \text {. }
$$

The amplitude of coherent oscillation in that case will be about

$$
A_{c} \simeq \Delta X_{s} *\left(N_{d}\right)^{0.5} \simeq 0.2 \mu \mathrm{m} \text {. }
$$

With feedback, the external noise strength requirements become less severe. The maximum external noises that the strongest feedback with gain $H_{n}=1$ can damp to the required level in coherent oscillations is equal to $\Delta X_{\max } \simeq \Delta X_{s}{ }^{*} N_{d}=7 \mu \mathrm{m}$ (compare with Eqs. (39) or (40) with $H_{n}=0$ and $H_{n}=1$ ).

Such damping requires a BPM pickup a with noise level at a few first harmonics of $\Delta X_{p} \simeq \Delta X_{\max } /$ $(2 n h)^{0.5}=5 \mu \mathrm{m} /(n h)^{0.5}$ ( $n h$ is number of harmonics in the feedback band). For damping of the resistive wall instabilities one needs $n h=100$, then the pickup noise should be less then $0.5 \mu \mathrm{m}$. The discussion in paragraph 3.4 of this section indicates that it is not a difficult task because the estimated pickup noise is only about $0.001 *\left(\mathrm{n}_{\mathfrak{n}}\right)^{0.5} \simeq 0.01 \mu \mathrm{m}$ in this frequency band. 
One can estimate the spectral power of single quadrupole vibrations $S_{s}$ that the highest-level feedback system can damp to the needed level:

$$
S_{s} \simeq\left(\Delta X_{\max }=7 \mu \mathrm{m}\right)^{2} /\left(N_{\text {quads }} \simeq 10^{3}\right) /\left(\Delta \mathrm{f} \simeq 10^{3} \mathrm{~Hz}\right)=5 \times 10^{-5} \mu \mathrm{m}^{2} / \mathrm{Hz} .
$$

In the absence of the feedback, the dangerous value of spectral power density of each quad vibrations (see Section 1) is equal to

$$
S_{p}<3 \times 10^{-12} \mu \mathrm{m}^{2} / \mathrm{Hz} \text {. }
$$

According to our analysis (Section 1) the values due to ground motion of such vibration spectra at $1000 \mathrm{~Hz}$ is about $10^{-12} \mu \mathrm{m}^{2} / \mathrm{Hz}$ in "quiet" conditions, $10^{-10} \mu \mathrm{m}^{2} / \mathrm{Hz}$ in "noisy" conditions, and about $10^{-8} \mu \mathrm{m}^{2} / \mathrm{Hz}$ for "real accelerator conditions" (see Figure 2).

For the following estimates the power of external noise takes the form:

$$
S_{0}(f)=10^{-7}(1.0 \mathrm{kHz} / f)^{4} \mu \mathrm{m}^{2} / \mathrm{Hz} .
$$

Corresponding spectrum for a single quad is about $10^{3}$ times less and the amplitude of a random turn-toturn kick is equal to $0.03 \mu \mathrm{m}$; therefore, the SSC luminosity lifetime without feedback is 40 minutes.

Figure 8 shows optimal feedback calculations according to Eqs. (38)-(42). Spectra of external noise and pickup noise are shown in Figure 8a. Figure $8 \mathrm{~b}$ presents the optimal feedback gain vs. frequency and Figure $8 \mathrm{c}$ shows amplitudes of coherent betatron oscillations for different harmonics with and without optimal feedback.

Figure $8 \mathrm{c}$ shows that when the feedback is switched off, the amplitude of coherent motion on first harmonics is too large-about 1 micrometer-but the feedback strongly damps that amplitude to $0.02 \mu \mathrm{m}$.

We should note that such a large value of the gain (about 1.0 at the first harmonic) requires one carefully choose the feedback phase characteristics taking into account the response functions of the beam and feedback loop. Otherwise, instabilities might occur as described in paragraph 3.2 above. Notch or digital filters may be used for phase correction of the feedback.17,18,19
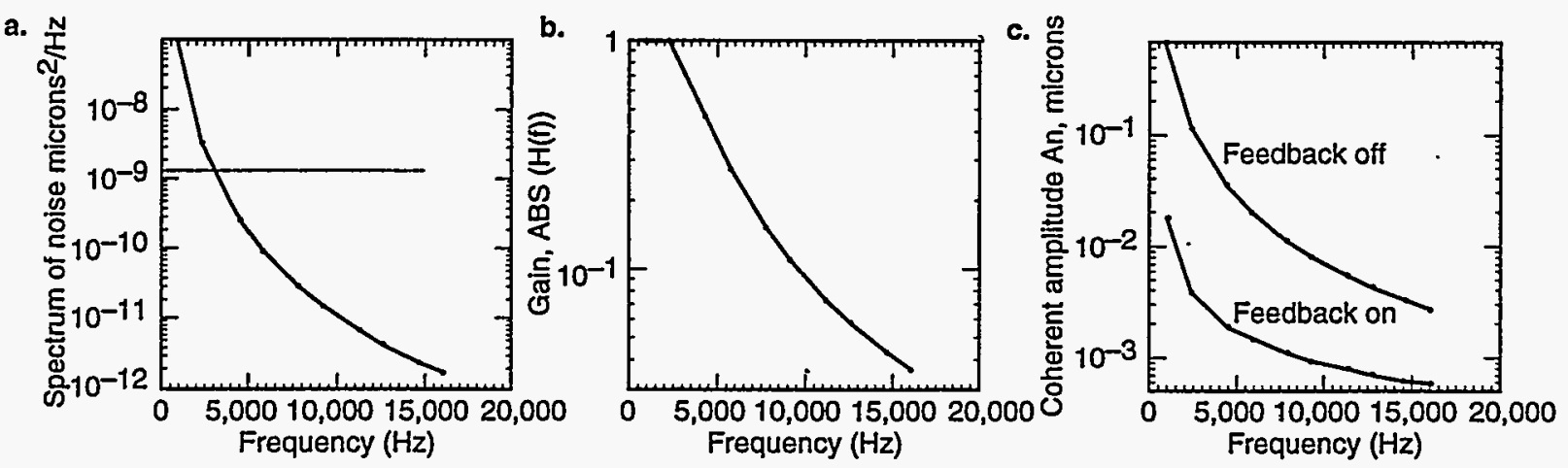

Figure 8. a) Spectrum of External Noise; b) An Optimal Feedback Gain vs. Frequency; c) Amplitudes of Coherent Betatron Oscillations for Different Modes-Feedback off, Feedback on. 


\subsection{General Scheme of the SSC Damping System}

Figure 9 shows a general scheme for a beam coherent oscillation damping system.

The BPM signal passes through a passive filter to the system that forms the amplitude-phase characteristics. Such a system includes analog and digital parts that form an optimal response function that damps beam oscillation at all harmonics.

The system also contains a second part that corrects the slow changing of the closed orbit at the BPM position. Usually the COD is a few orders of magnitude greater than the beam noise on resonance frequencies. Without COD correction the damping system may reduce the amplitude range of the fast kicker.

One way uses the average beam position and controls slow orbit motion by using several slower dipole correctors. Another possibility is to mechanically move the BPM for the BPM center should be positioned exactly on the average position of the closed orbit. ${ }^{20}$ The latter seems better in that the correction system is localized and does not interfere with other orbit correction systems.

Additional development work will help determine what kind of correction is best for the SSC.

The feedback kicker and amplifier power needed depend on the amplitude of the proton beam noise. For example, if one wants to damp $\Delta X=1 \mu \mathrm{m}$ oscillations of the beam, the kicker must produce angular proton beam deviations of about $\Delta \vartheta=3 \times 10^{-9}$. $\mathrm{rad}$ (because the value of the beta function in the kicker is about 200 meters). Such a corrector with a length $l=1 \mathrm{~m}$ and an aperture $a=10 \mathrm{~cm}$ will need a certain amount of energy $\delta W$ of electric (or magnetic) field $E$ :

$$
\delta W=\frac{E^{2}}{8 \pi} \pi l a^{2} \simeq \Delta X^{2}(\mu \mathrm{m}) a^{2}(\mathrm{~cm}) / l(\mathrm{~cm}) * 4 \times 10^{-4}[\mathrm{~J}]=4 \times 10^{-4} \mathrm{~J} .
$$

For a narrowband feedback system with $\Delta f \approx 100 \mathrm{kHz}$, the necessary amplifier power is about 40 Watts. But if one uses feedback with a bandwidth of about $60 \mathrm{MHz}^{21}$ then the needed power will be $24 \mathrm{~kW}$ (this is not simple task)!

The real parameters of the damper system should be chosen after a more detailed investigation of main components: low noise BPM, the correction system, the filter, the feedback amplifier, and kicker.

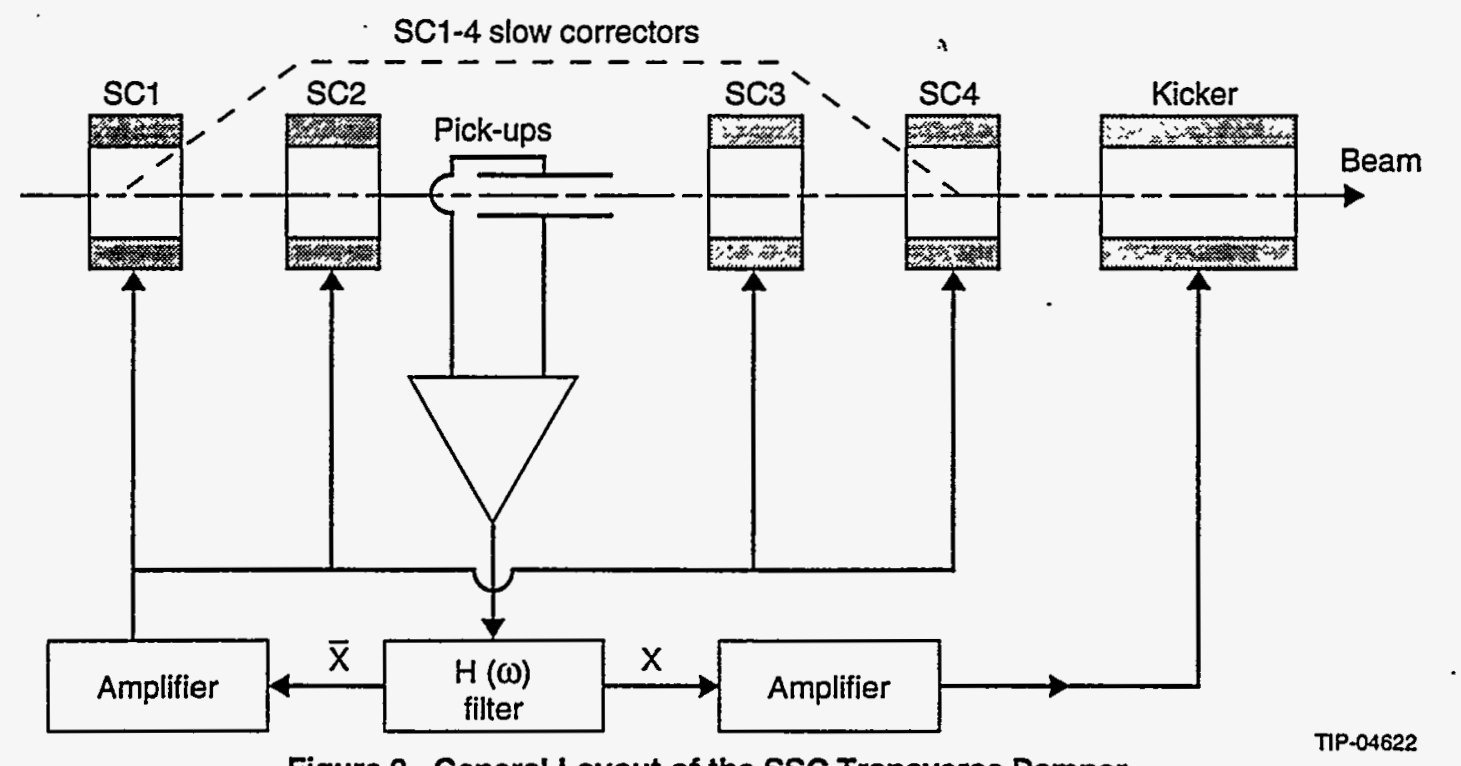

Figure 9. General Layout of the SSC Transverse Damper. 


\subsection{MAIN CONCLUSIONS}

We summarize the preceding discussions as follows:

1. The levels of measured ground vibrations under the special very quiet conditions are only marginally acceptable for the SSC quadrupoles high frequency vibration. Measured levels of magnet vibrations under conditions which closely resembled real conditions (but without full cryogenic environmental noises) exceed dangerous values.

Data from operating accelerators show that one must expect a further increase of vibrations during the collider operation.

2. Our preliminary measurements and analysis of worldwide data indicate another possible strong source of beam "shaking." It is the turbulent flow of cooling liquid and/or gas inside the magnetic elements. If the strength of the effect is comparable with other accelerator observations, the SSC emittance lifetime will be in the minute range.

3. The transverse beam-based feedback system is a practical way to prevent emittance decay and keep collider luminosity. Parameters of the feedback depend on parameters of external noise as well as beam dynamics in the SSC. In addition to the feedback, all other reasonable ways to mechanically decrease vibrations should be used at the collider.

4. Ambient coherent betatron oscillations produce emittance growth due to decoherence processes. Minimizing coherent oscillation amplitude reduces transverse emittance growth. Selecting optimal values of transverse feedback gain and frequency band minimize betatron oscillation amplitude. At optimal parameters, coherent oscillations are excited at the same values whether by external noise or by proper noises of the feedback (noises of BPM and kicker, digital noises, etc.)

5. Optimization shows that the collider transverse feedback system should have a narrow frequency band (similar to external noises frequency band-about a dozen $\mathrm{kHz}$ ). To achieve the necessary emittance growth rate reduction with an optimal feedback system there are some slight requirements for the feedback elements: for example, the pickup noises have to be small only at low frequencies (i.e., below a dozen $\mathrm{kHz}$ compared with the SSC collision frequency of $60 \mathrm{MHz}$ ).

\section{ACKNOWLEDGMENTS}

The authors acknowledge discussions with Brett Parker, Rainer Meinke and Edward Tsyganov. We are grateful to Valery Lebedev for reading the manuscript and his useful comments. 


\section{REFERENCES}

1. V. A. Lebedev, V. V. Parkhomchuk, V. D. Shiltsev, and A. N. Skrinsky, "Suppression of Emittance Growth Caused by Mechanical Vibrations of Magnetic Elements in Presence of Beam-Beam Effects in the SSC," Preprint INP 91-120, Novosibirsk, 1991.

2. V. A. Lebedev, V. V. Parkhomchuk, V. D. Shiltsev, and G. V. Stupakov, "Emittance Growth due to Noise and Its Suppression with the Feedback System in Large Hadron Colliders," SSCL-Preprint188, March 1993.

3. V. A. Lebedev, "Computer Simulation of the Emittance Growth Due to Noise in Large Hadron Colliders," SSCL-Preprint-191, March 1993.

4. G. E. Fischer and P. Morton, "Ground Motion Tolerances for the SSC," SSC-55, 1986.

5. K. Y. Ng and J. O. Peterson, "Ground Motion Effects on the SSC," SSC-212, 1989.

6. V. V. Parkhomchuk, V. D. Shiltsev, and H. J. Weaver, "Measurements of the Ground Motion and the SSC Dipole Vibrations," SSCL-Report-624, April 1993, in print.

7. V. V. Parkhomchuk and V. D. Shiltsev, "Fractal Model of the Ground Motion," Preprint INP 92-31, Novosibirsk, 1992.

8. B. A. Baklakov, P. K. Lebedev, V. V. Parkhomchuk, A. A. Sery, V. D. Shiltsev, and A. I. Sleptsov, "Investigation of Seismic Vibrations and Relative Displacement of Linear Collider VLEPP Elements," Proc. of 1991 IEEE Part. Accel. Conf., San Francisco, USA, p. 3273, May 1991.

9. W. Decking, K. Floetman, and J. Rossbach, "Measurement of Slow Closed Orbit Motion in Correlation with Ground Motion," Proc. of II European Part. Acc. Conf., Nice, March 12-16, 1990, pp. 1449-1451, and DESY-M-90-02, Feb.1990.

10. V. A. Lebedev, P. K. Lebedev, V. V. Parkhomchuk, and V. D. Shiltsev, "Transverse Vibrations of Electron Beam and Ground Motion Measurements at VEPP-3 Storage Ring," INP Preprint 92-39, Novosibirsk, 1992, and Proc. of 1992 Int. Part. Acc. Conf., Dubna, Russia, October 1992.

11. Y. Kobayashi et al., "A Test of 3-GeV Operation at the Photon Factory Storage Ring," Proc. of III European Part. Acc. Conf., Berlin, March 24-28, 1992, pp. 3-7.

12. R. P. Johnson and P. Zhang, "A New Tevatron Collider Point Near the Integer," Proc. of IEEE Part. Accel. Conf., Chicago IL, March 20-23, 1989, pp.806-808.

13. D. A. Herrup et al., "Luminosity Lifetime in the Tevatron Collider," Proc. of IEEE Part. Accel. Conf., Chicago IL, March 20-23, 1989, pp.1823-1826.

14. D. Degele, "The e-p Collider HERA," Proc. of III European Part. Acc. Conf., Berlin, March 24-28, 1992, pp. 3-7.

15. B. A. Baklakov, P. K. Lebedev, V. V. Parkhomchuk, and V. D. Shiltsev, Proposal "Vibration Measurements for the SSC," INP, Novosibirsk, May 1992 (not published).

16. D. Briggs, "Low Frequency Transverse Resistive Wall Instability in the Collider," SSCL-512, June 1992.

17. R. Cappi, G. Lumbertson, and L. Vos, "Wide-Band Feedback for Hadron Supercolliders," Workshop on Feedback Systems, Erice, Italy, Nov. 13-21, 1992.

18. W. Chou and J. Peterson, "The SSC Transverse Feedback Systems," Workshop on Feedback Systems, Erice, Italy, Nov. 13-21, 1992.

19. Proc. of Workshop "B-Factories: The State of the Art in Accelerators, Detectors and Physics," SLAC, April 6-10 1992, Part "Feedback System," pp.192-223, SLAC-400, Nov. 1992.

20. Brett Parker, private communication.

21. E. Tsyganov et al., "The SSC Damper System," SSCL-Preprint-202, March 1993. 